\title{
Wie Sie bei Hochdruckpatienten den „Hyper-Aldo“ abklären
}

\author{
Wird ein Hyperaldosteronismus frühzeitig erkannt, lässt er sich in jedem zweiten Fall heilen. \\ In der neuen Leitlinie der Endocrine Society ist geregelt, welche Hochdruckpatienten man \\ screenen sollte und welche Verfahren dabei zum Einsatz kommen.
}

_ Die häufigste Ursache einer sekundären Hypertonie ist der Hyperaldosteronismus (auch: primärer Aldosteronismus, PA). Nach Prof. Martin Fassnacht vom Uniklinikum Würzburg betrifft dieser zwischen 4 und 12\% aller Hypertoniker. Werde die Diagnose frühzeitig gestellt, könne man etwa jeden zweiten $\mathrm{Pa}$ tienten heilen. Ansonsten hätten die Betroffenen bei gleichen Blutdruckwerten ein deutlich schlechteres Outcome als $\mathrm{Pa}$ tienten mit essenzieller Hypertonie.

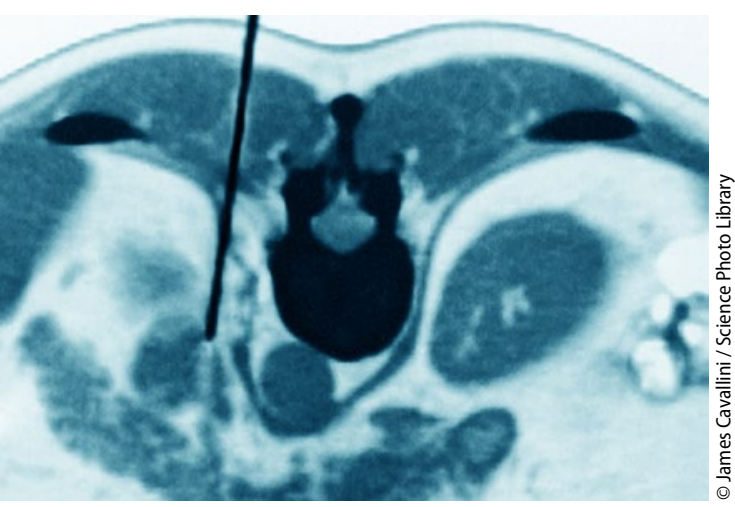

Aspirationsbiopsie eines Nebennierentumors.

\section{Wen screenen?}

Wann ein Screening auf Hyperaldosteronismus durchgeführt werden sollte, wird durch eine neue Leitlinie ${ }^{\star}$ geregelt. Für Fassnacht relevant sind dabei vor allem folgende Voraussetzungen:

*Funder JW et al. The Management of Primary Aldosteronism: Case Detection, Diagnosis, and Treatment: An Endocrine Society Clinical Practice Guideline. J Clin Endocrinol Metab 2016; 101: 1889-916

http://press.endocrine.org/doi/pdf/10.1210/jc.2015-4061
- Vorliegen einer Hypertonie mit Werten > 140/90 mmHg unter Einnahme von drei Antihypertensiva,

- Hypertonie und Hypokaliämie,

- Hypertonie und Nebennierenraumforderung.

„Je jünger der Patient, desto sinnvoller ist es, an einen PA zu denken“, so Fassnacht.

\section{Wie screenen?}

Laut Fassnacht sind Patienten mit Hyperaldosteronismus i.d. R. normokaliämisch. „Daher ist es komplett obsolet, das Kalium als Screening-Parameter zu verwenden“. Die Leitlinie empfiehlt die Bestimmung des Aldosteron/Renin-Quotienten (ARQ). Für die Blutabnahme sollten idealerweise mindestens zwei Stunden nach dem Aufstehen vergangen sein und der Patient sollte zuvor mindestens fünf $\mathrm{Mi}$ nuten sitzen. Wichtiger ist nach Fassnacht das vierwöchige Pausieren von Aldosteronantagonisten wie Spironolacton und Eplerenon, aber auch z.B. von Drospirenon-haltigen „Antibabypillen“: „Unter dieser Therapie können Sie keine saubere Diagnostik machen." Andere Blutdrucksenker könne man weiterlaufen lassen, wenn man wisse, wie sie den ARQ möglicherweise beeinflussen. Eine entsprechende Aufstellung gibt die Leitlinie*.

Ein grenzwertiger Befund im ARQ sollte Anlass sein, die Blutdruckmedikamente umzustellen. „Neutrale“ Medikamente sind in dieser Hinsicht z. B. Verapamil, Dihydralazin oder Alphablocker.

\section{Absicherung der Diagnose}

Bei positivem Testergebnis ist aufgrund der relativ geringen Spezifität bei den meisten Patienten ein Bestätigungstest nötig: „Wir wissen“, so Fassnacht, „dass viele Patienten mit erhöhtem Quotienten keine Erkrankung haben." Als Standard gilt der Kochsalzbelastungstest (2 Liter $0,9 \% \mathrm{NaCl}$ über $4 \mathrm{~h}$ infundieren). Ist dieser pathologisch, d.h. bei fehlender Plasma-Aldosteron-Suppression mit Werten $>10 \mathrm{ng} / \mathrm{dl}$, folgt als nächstes eine CT der Nebenniere. Diese, so Fassnacht, gebe zwar Hinweise darauf, ob es sich um einen uni- oder bilateralen Hyperaldosteronismus handle. Gesichert werden könne dies jedoch nach wie vor am besten über die selektive Blutentnahme aus der Nebennierenvene (NNV-Katheter). Nur wenn dieses Verfahren die unilaterale Erkrankung bestätige, sei eine Operation in Form einer laparoskopischen Adrenalektomie indiziert. Andernfalls werde mit Spironolacton oder Eplerenon behandelt.

\section{Bestätigungstest im Einzelfall verzichtbar!}

Als sinnvolle Ausnahmeregelungen nannte Fassnacht zwei Neuerungen: So könne man bei komplett supprimiertem Renin, zusätzlich bestehender Hypokaliämie und „halbwegs“ (> 20 ng/dl) erhöhtem Aldosteron am Bestätigungstest vorbei direkt zur nächsten Stufe gehen. Die zweite Ausnahme betrifft Patienten unter 40 Jahren mit spontaner Hypokaliämie und ausgeprägtem Aldosteronexzess, bei denen die CT eine unilaterale Nebennierenraumforderung zeigt. „Diese“, so Fassnacht, „können auf den selektiven NNV-Katheter verzichten und direkt zur Op. gehen."

\section{Dr. Elke Oberhofer}

- Internisten-Update, 18./19. November 2016 in München 